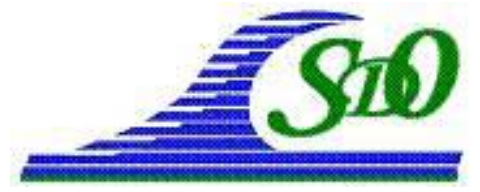

XI ${ }^{\text {èmes }}$ Journées Nationales Génie Côtier - Génie Civil

Les Sables d'Olonne, 22-25 juin 2010

DOI:10.5150/jngcgc.2010.101-F @ Editions Paralia CFL

disponible en ligne - http://www.paralia.fr - available online

\title{
Restructuration du port de la Rague : une gestion environnementale exemplaire
}

\author{
Pierre FARNOLE ${ }^{1}$, Jean-Pierre BLANC ${ }^{2}$
}

1. ERAMM, 1503 rte des Dolines, BP 42, 06901 Sophia Antipolis, France.

pf@eramm.fr

2. OMEGA Consultants, France.

jpb@omegaconsultants.fr

\section{Résumé :}

Le port de la Rague est constitué d'un petit et d'un grand bassin qui offre une capacité d'amarrage de 424 bateaux de 5 à $40 \mathrm{~m}$ de longueur et d'un port à sec de 70 places. Il est précurseur dans les Alpes-Maritimes par les actions qu'il a entreprises tant sur le volet environnemental que sur sa gestion et sur l'optimisation de ses installations. Ainsi, le port de la Rague a réalisé son diagnostic environnemental dès 2004 et acquis la normalisation 030 - Gestion environnementale portuaire en 2009 reconduite en 2010. Dès 2004, le port de la Rague a entrepris des travaux de restructuration avec l'aménagement de l'avant-port pour l'accueil de 7 grandes unités de plaisance de 20 à $40 \mathrm{~m}$ et l'extension de son chantier naval dans le petit bassin qui s'est achevé en 2006 avec mise aux normes des installations. En 2007, le Port de la Rague, avec le concours des sociétés co-concessionnaire, a poursuivi sa politique de mise en valeur et d'optimisation du port par la création sur terrain hors concession, d'un port à sec pouvant accueillir 70 petites unités. Il souhaite maintenant créé un nouveau bassin pour la moyenne et grande plaisance répondant ainsi à l'absence d'offre dans ces catégories et à la forte demande actuelle dans le bassin mandelocien.

\section{Mots-clés :}

Port propre - Diagnostic environnemental - Restructuration portuaire - Economie portuaire - Développement durable - Port de plaisance

\section{Introduction}

Le Port de La Rague, situé entre les communes de Mandelieu-la-Napoule et Théoulesur-Mer, créé en juillet 1969, est un port de plaisance constitué d'un petit et d'un grand bassin qui offrait en 2004 une capacité d'amarrage de 520 bateaux de 5 à $40 \mathrm{~m}$ de longueur (figure 1). Les sociétés co-concessionnaires (Société d'Exploitation du Port de la Rague et Société Nautique et Balnéaire du port de la Rague) ont eu la volonté de moderniser la gestion du port et d'accroître sa rentabilité mais également de satisfaire aux contraintes européennes et françaises en matière de protection de l'environnement. Le port de la Rague a procédé à des travaux de restructuration avec l'aménagement de l'avant-port pour l'accueil de 7 grandes unités de plaisance de 25 à $40 \mathrm{~m}$ et l'extension 
de son chantier naval dans le petit bassin qui a été achevée en 2005. Le port de la Rague a entrepris, dès 2004, la mise aux normes environnementales par la réalisation d'un diagnostic environnemental dont les actions sont aujourd'hui en grande partie réalisées. Dans la poursuite de cette démarche de mise aux normes environnementales, le port a acquis la certification: ACJ81 - 030 - Gestion environnementale portuaire en 2009 reconduite en 2010. Sur le volet énergétique, le port a pris des dispositions pour réduire sa consommation énergétique sur l'ensemble du port.

Parallèlement à cette normalisation, le port de la Rague a procédé à des travaux de restructuration avec l'aménagement de l'avant-port pour l'accueil de quelques grandes unités de plaisance de 20 à $40 \mathrm{~m}$ et l'extension de son chantier naval dans le petit bassin. Ces travaux, bien que conséquents, n'ont pas permis de satisfaire la demande très forte des plaisanciers pour des unités de moyenne et grande plaisances qui n'ont plus de possibilité d'accueil dans le golfe de la Napoule. Devant le succès de l'opération de restructuration achevée en 2006, le Port de la Rague souhaite poursuivre sa restructuration en créant un nouveau bassin pour 100 unités de moyenne et grande plaisances répondant ainsi à la forte demande actuelle.

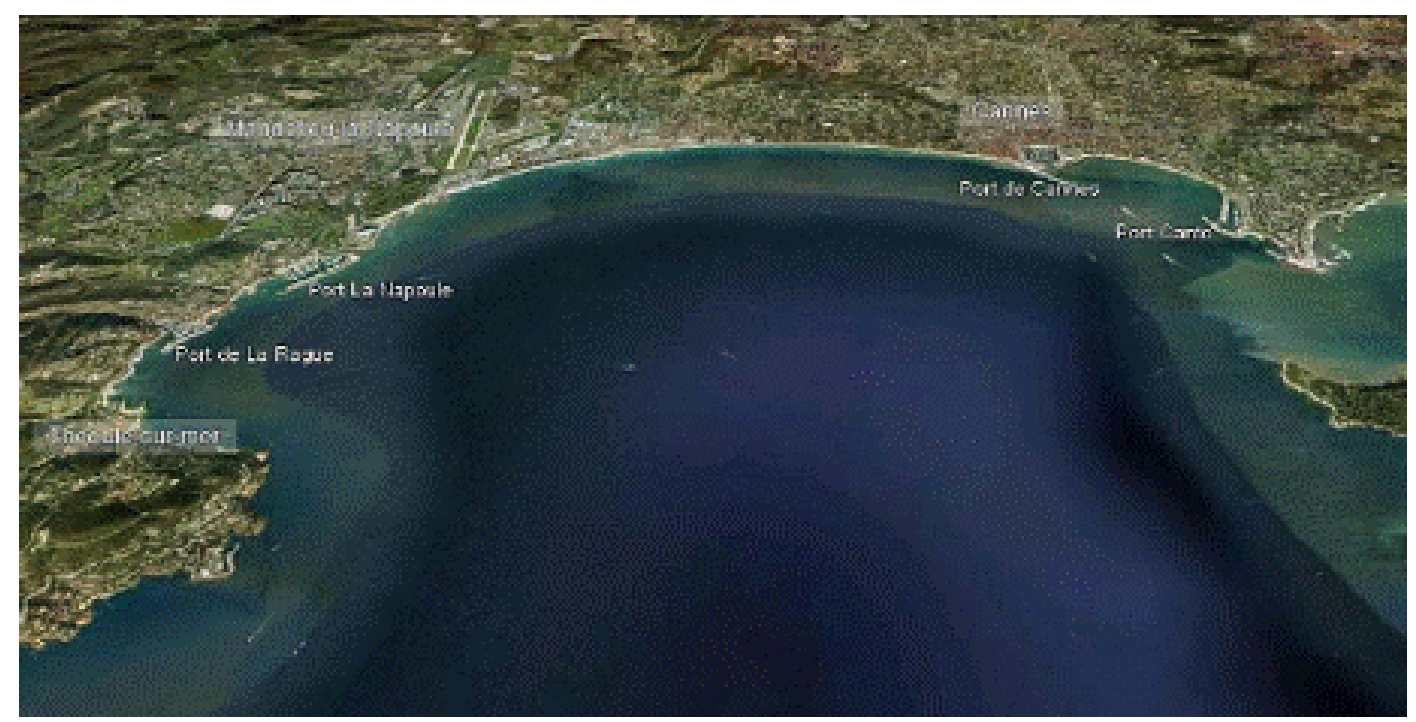

Figure 1. Vue aérienne verticale du golfe de la Napoule.

\section{Profil économique du port}

A l'heure actuelle, on dénombre 9 structures directement liées au port, et implantées dans le périmètre portuaire. En termes économiques, la filière, dans son ensemble, représente une activité estimée à $10 \mathrm{M} €$ et génère près de 60 emplois (ETP) pour une masse salariale de 2 M€ (tableau 1). Le port de La Rague est un véritable outil économique en termes d'impacts qualitatifs et quantitatifs mais aussi un acteur socioéconomique important, 60 ETP directs (dont la majorité des dépenses induites sont situées sur la commune ou les communes limitrophes) et 198 ETP indirects. 
Tableau 1. Filière économique du port de la Rague.

\begin{tabular}{llllll}
\hline Structures & Nombre & C.A. $($ en $\boldsymbol{K} \boldsymbol{E H T})$ & $\boldsymbol{E T P}$ & $\begin{array}{l}\text { Masse salariale } \\
(\text { en K€ HT) }\end{array}$ \\
\hline Port & 1 & 1559 & $16 \%$ & 13,5 & 606 \\
Clubs et associations nautiques & 3 & 200 & $2 \%$ & 3,25 & 97,5 \\
Entreprises du nautisme & 5 & 8000 & $82 \%$ & 42 & 1356 \\
Pêche professionnelle & 1 & - & - & 1 & - \\
TOTAL & 10 & 9799 & $100 \%$ & 59,75 & 2059 \\
\hline
\end{tabular}

\section{Profil environnemental du Port}

\subsection{La démarche "port propre" en Région Paca}

La démarche "Port Propre en Région PACA" a été entreprise par le Port de la Rague dès 2004 par l'élaboration d'un diagnostic environnemental dont les objectifs sont :

- de définir la présentation géographique, administrative et juridique du port, et de son environnement associé,

- de donner des informations précises sur les origines, la nature, et l'impact des nuisances recensées sur l'environnement,

- de réaliser une description exhaustive des solutions et des équipements portuaires et de gestion des déchets déjà existants,

- de lister les objectifs de qualité souhaités pour le plan d'eau, les sédiments et les installations à terre.

L'aboutissement de ce diagnostic a conduit à l'élaboration d'un programme d'actions pluriannuelles et un calendrier de réalisation des travaux dans l'objectif d'obtenir le label "port propre en Région PACA". Le port de la Rague a entrepris, dès 2004, la mise aux normes environnementales par la réalisation d'un diagnostic environnemental dont les actions sont aujourd'hui en grande partie réalisées :

- Extension et mise à niveau de la station d'avitaillement en 2005 (figure 2) ;

- Extension et mise à niveau de l'aire de carénage en 2006 (figure 3) ;

- Réfection du réseau d'assainissement en 2007 ;

- Formation du personnel à la gestion environnementale : 2 agents depuis 2007 ;

- Création d'un bloc sanitaire en 2008 ;

- Traitement des terre-pleins des quais 6-7-8 en 2009;

- Installation du "Point propre" et tri sélectif des déchets en 2009-2010;

- Mise à niveau du kit antipollution pour lutter contre la pollution accidentelle en 20092010 ;

- Récupération des eaux usées, grises et noires et des eaux de fond de cale en 2011. 


\subsection{La Certification Afnor}

Dans la poursuite de cette démarche de mise aux normes environnementales, le port a acquis la certification AFNOR : ACJ81 - 030 - Gestion environnementale portuaire en 2009. Le référentiel "ACJ81 - 030 Gestion environnementale portuaire" porte sur différents critères spécifiques aux problématiques portuaires : gestion des déchets et des pollutions, information et écoute des usagers, gestion des pollutions accidentelles, formation du personnel, gestion des ressources en eau et énergétique. Cette certification ne peut être sollicité que si l'organisme demandeur a effectué le diagnostic environnemental et s'il a déjà engagé le programme d'actions avec les travaux de mise aux normes (cas du port de la Rague dès 2008). Il a pu ainsi acquérir cette certification qui a été reconduite en 2010 après un audit de contrôle de l'Afnor.

Sur le volet énergétique, le port a pris des dispositions pour réduire sa consommation énergétique qui est effective en période estivale.

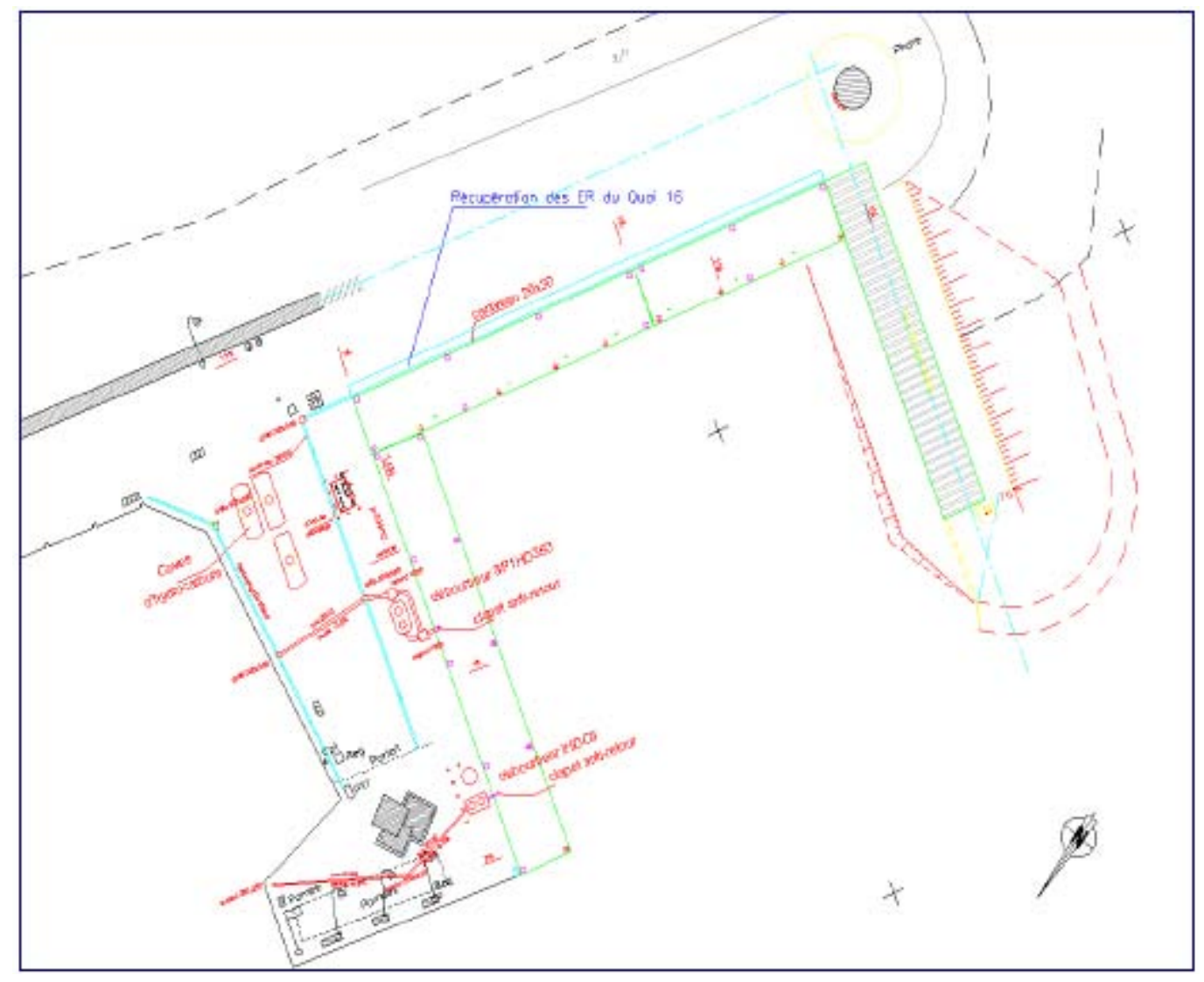

Figure 2. Extension et mise à niveau de l'avant-port. 


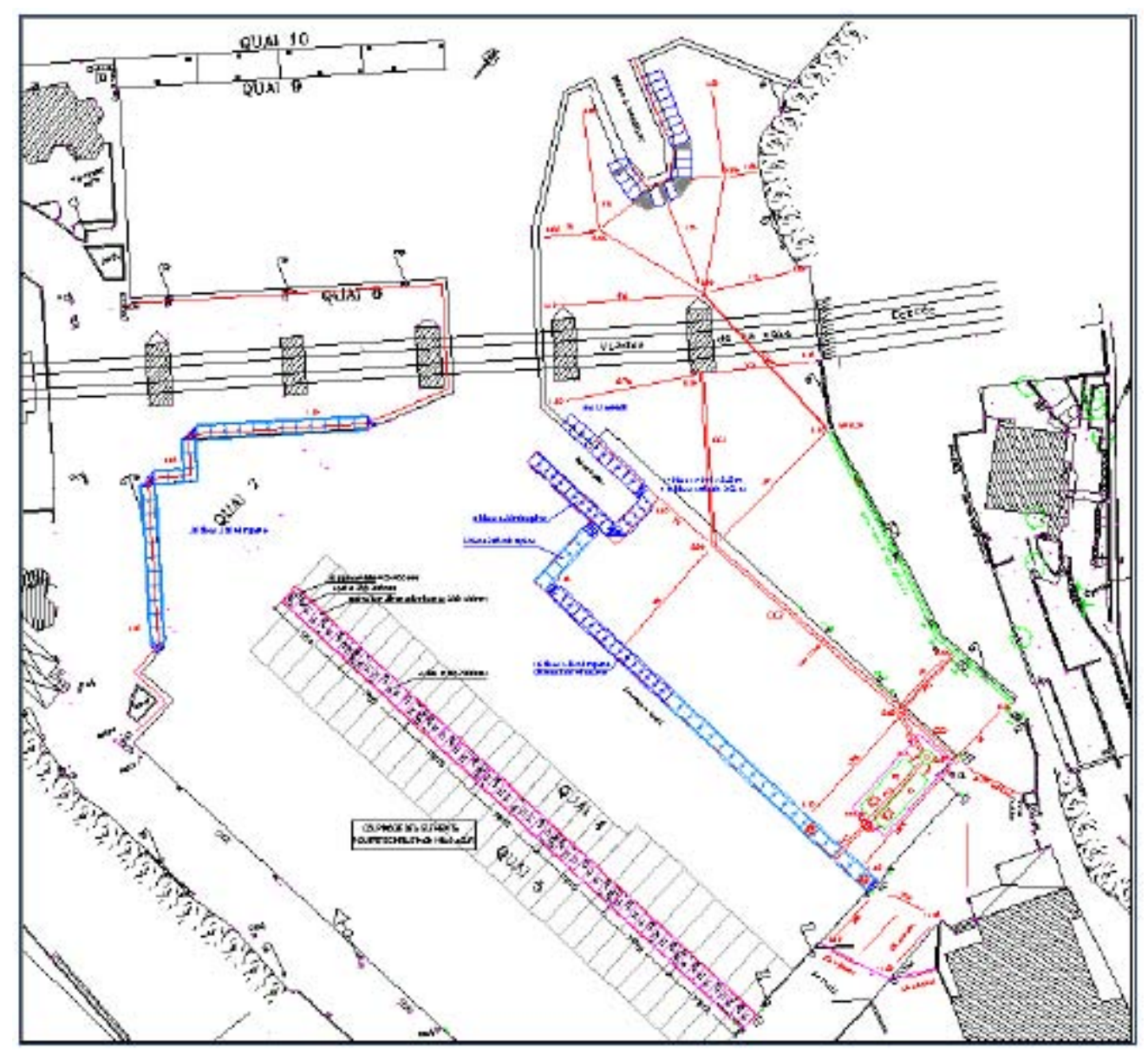

Figure 3. Extension et mise à niveau de l'aire de carénage.

\section{Projet de création d'un nouveau bassin}

Le projet d'extension est un projet de développement économique mais c'est aussi un projet urbain qui s'inscrit dans une volonté de rapprocher le port de la Rague des ports et des centres-urbains de Cannes, Mandelieu-la-Napoule et de Théoule-sur-Mer, en y installant un transport urbain maritime en période estivale. Les navettes à moteur hybrides constitueront un moyen de déplacement rapide, non polluant et agréable car elles permettront d'offrir un autre regard du littoral de la mer vers la terre. Ce projet s'inscrit donc comme un véritable service au public qui n'existe pas actuellement entre les ports et les villes dans l'ouest du département. Pour faciliter les accès terrestres, un minibus sera mis en service entre les différents points du port depuis son entrée jusqu'à l'appontement des navettes. L'innovation majeure en matière d'environnement réside dans la structure de la digue du port qui est de type digue partielle mixte qui lui confère une quasi transparence en termes de circulation des eaux entre baie et port. Elle présente l'avantage de minimiser l'emprise sur le fond et à maintenir le flux entre le bassin portuaire et la mer. Cette technique permet une meilleure intégration paysagère en $y$ insérant des blocs en enrochements de couleur rouge de l'Estérel. L'impact sur les 
plages adjacentes sera minimisé par rapport à un ouvrage massif car une partie de l'énergie de la houle sera dissipée aux extrémités réduisant ainsi les phénomènes de diffraction-radiation qui, dans bon nombre de cas, affectent les plages.

\section{La préservation de l'environnement}

Le projet innovant en matière environnementale est d'atteindre une totale autonomie en matière d'assainissement et une réduction significative de la consommation énergétique. Il est aussi innovant car il propose de faire du port un lieu de reconquête écologique par la transparence des digues à la circulation des courants et la valorisation des fonds marins par l'immersion de récifs artificiels :

- Digue semi-transparente à la circulation des courants pour favoriser les échanges entre le nouveau bassin et la mer (figure 4 et figure 5) ;

- Préservation des écosystèmes côtiers (herbiers de Posidonies et grandes nacres);

- Installation de récifs artificiels le long de la nouvelle digue pour repeupler les fonds marins (figure 4) ;

- Appontement pour les liaisons maritimes ;

- Station d'épuration intégrée avec comme objectif de "zéro rejet";

- Mouillages organisés à l'extérieur du port pour une vingtaine d'unités afin d'accroitre la capacité d'accueil des navires de passage ;

- Tri sélectif des déchets ;

- Panneaux photovoltaïques insérés sur les gardes au corps (figure 6) ;

- Une intégration dans le site (figure 7).

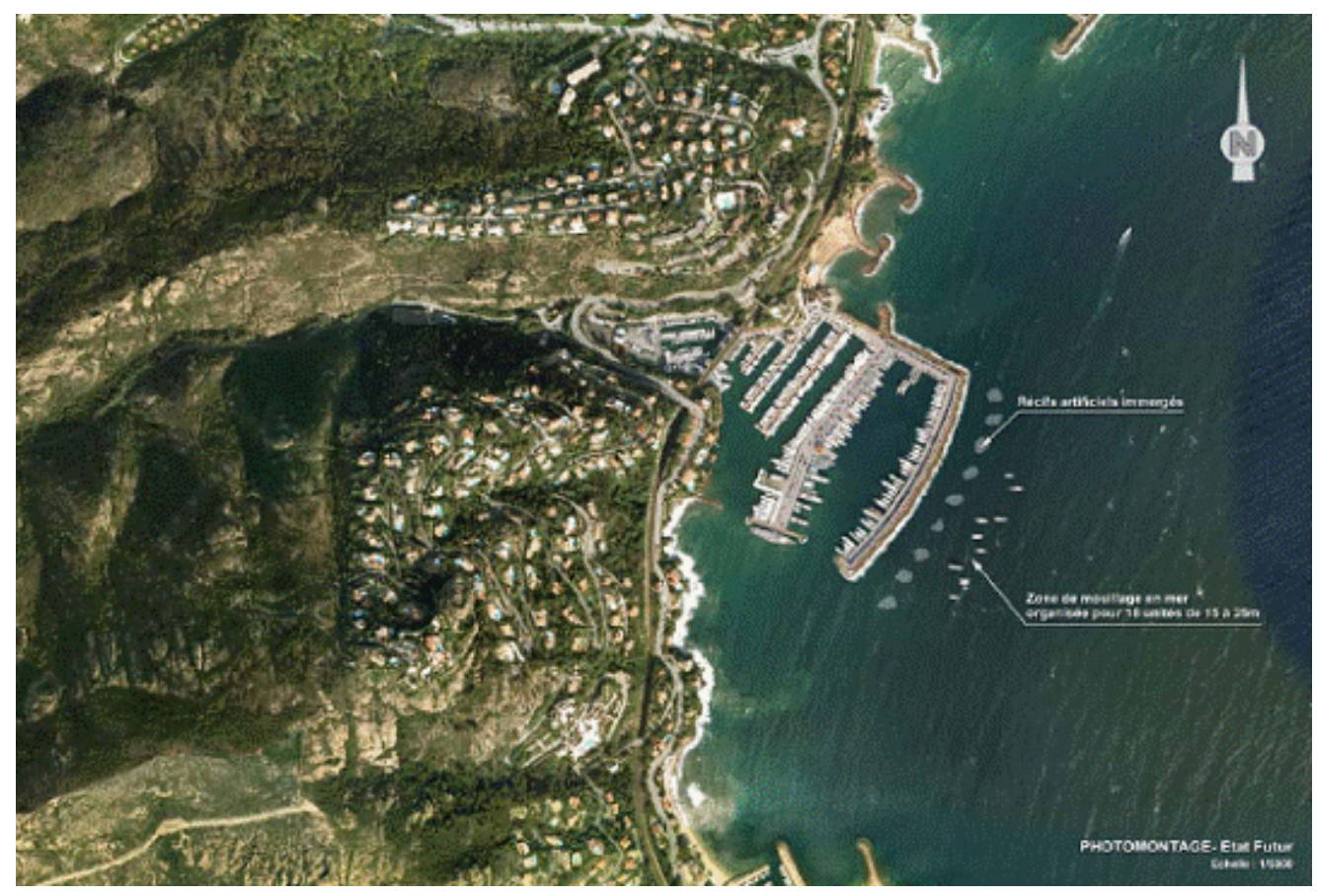

Figure 4. Vue aérienne du projet d'extension. 


\section{XI ìmes Journées Nationales Génie Côtier-Génie Civil}

Les Sables d'Olonne, 22-25 juin 2010
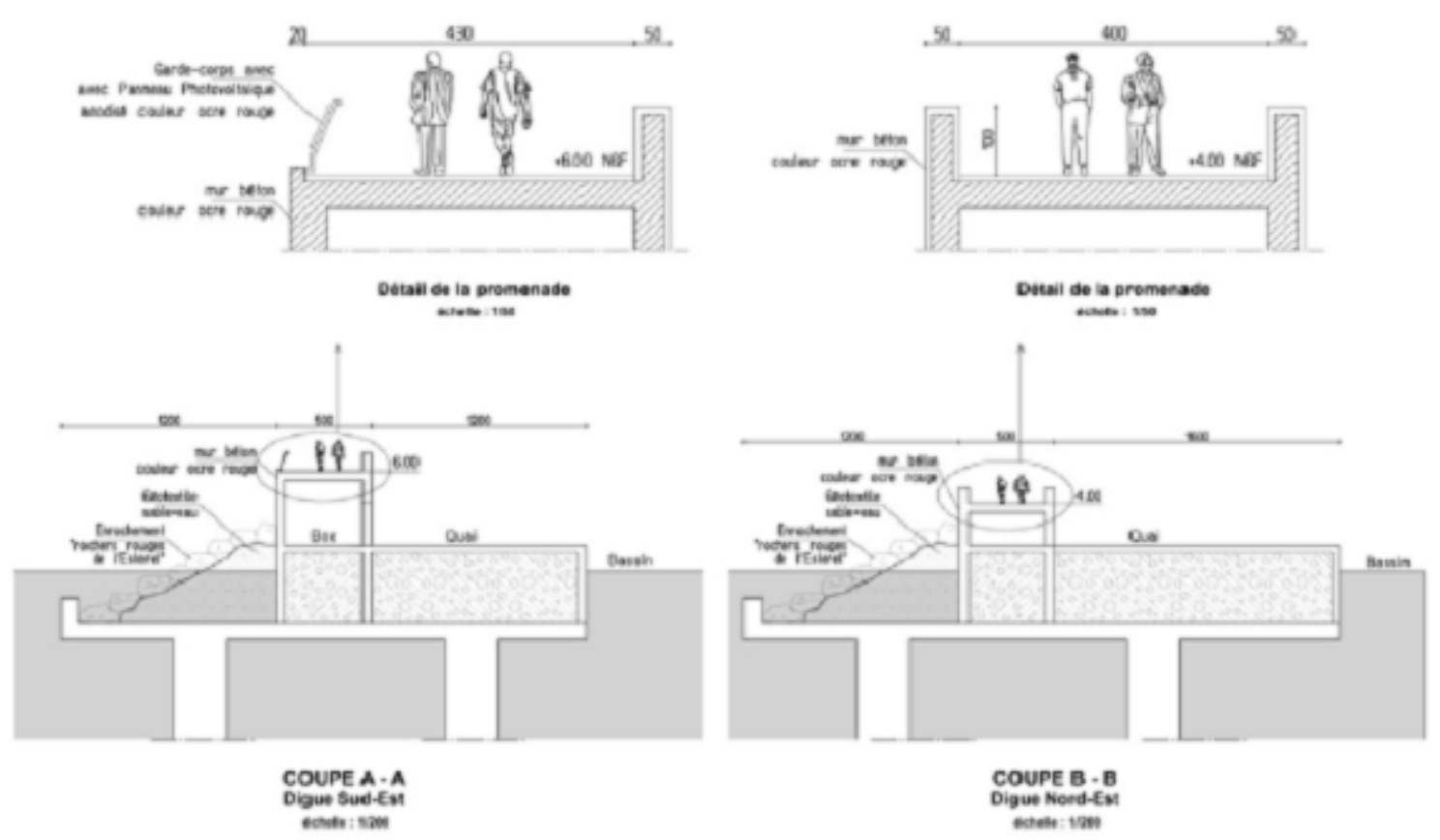

Figure 5. Coupes types de la digue du large.

COUPES A et B

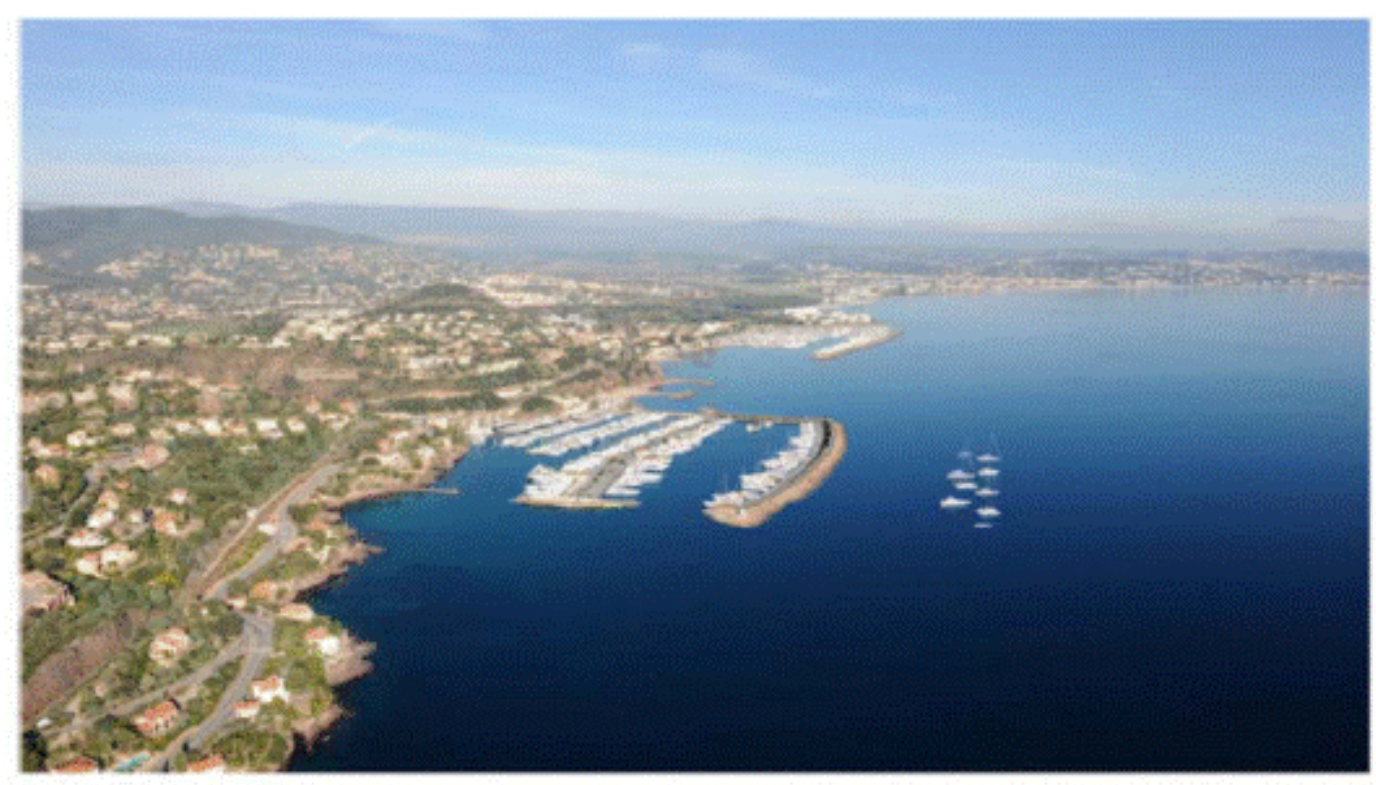

PHoromontace - Eat rubur

Figure 6. Implantation de panneaux photovoltaïques.

\section{Conclusions}

La mission publique des ports de plaisance évolue désormais vers une animation et un développement du territoire, basés sur des échanges touristiques, sur des nouvelles formes de mobilité (liaisons maritimes et sentier du littoral) mais aussi vers une 
sensibilisation de plus en plus forte de l'ensemble des usagers des ports de plaisance à la préservation des écosystèmes côtiers. Pour cela le port doit d'abord être exemplaire dans sa démarche environnementale mais il doit aller au-delà de cette démarche en évoluant vers le concept fort de "Port passif", c'est-à-dire avec zéro rejet dans le port et une autonomie en matière de consommation énergétique. Le port de La Rague s'oriente désormais vers un vrai concept de "station touristique portuaire" avec une parfaite maitrise de sa gestion environnementale.

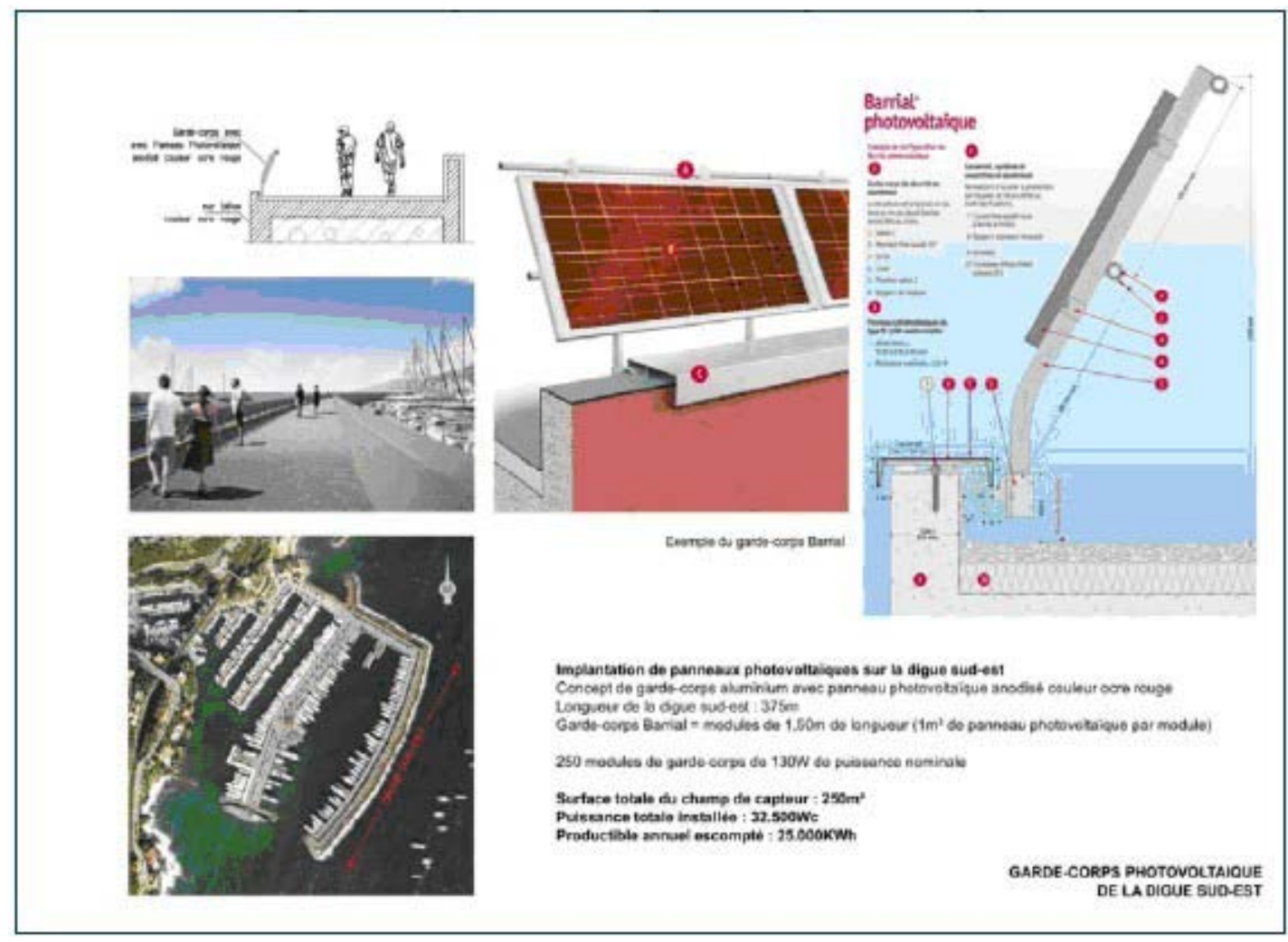

Figure 7. Photomontage d'après vues aériennes obliques.

\section{Remerciements}

Aux administrateurs et au personnel du port de la Rague et à la société Arie de Boom Marine.

\section{Références bibliographiques}

MEEDM (2010). Appel à projets pour des ports de plaisance exemplaires.

ERAMM (2004). Diagnostic environnemental du Port de la Rague.

ERAMM (2010). Appel à projets pour des ports de plaisance exemplaires : Extension du port de la Rague et création d'une zone de mouillage organisée.

OMEGA consultants (2009). Projet de restructuration du port de la Rague : étude socio-économique. 